\title{
Design alternatives for a thin lens spatial integrator array
}

\author{
Hala Kamal', Javier Alda'1, Daniel Vázquez¹, Eusebio Bernabéu² \\ 1 Optics Department, University Complutense of Madrid, School of Optics, Av. Arcos de Jalón s/n. 28037 Madrid, Spain \\ 2 Optics Department, University Complutense of Madrid, Faculty of Physics, Ciudad Universitaria s/n. 28040 Madrid, Spain
}

\begin{abstract}
In this paper we analyze different alternatives in the design of optimized optical arrays having spatial integration feature in a plane arrangement. More specifically, we focus our attention in the evaluation of the number of useful individual units, and its contribution to the synthetic image characteristics. The analysis has been made by using matrix optics relations. Two examples of planar thin lens spatial integrator arrays are designed and compared.
\end{abstract}

Key words: Optical arrays - spatial integrators - matrix optics - natural lighting

\section{Introduction}

Compared with a conventional optical system, where the light propagates through the components of the systems one after the other, in serie, an optical array processes the incoming light in parallel. It divides the incident wave front into portions which proceed through the individual optical elements to form a composed image. This image is called as the synthetic image, and, in general, it does not coincide with the image of the object given by the individual elements. This study is focused on an interesting type of optical arrays called spatial integrators. They are non-imaging devices and they are described by a ray transfer matrix having a null determinant. The essential characteristics of spatial integrators are an infinite depth of field, and a synthetic image size and location that do not depend on the location of the object. In other words, those rays coming from every light source in the object space, and arriving to the input plane of the array, are transferred to the synthetic image by the spatial integrator. Despite of they are non imaging devices, they have found several interesting applications. Typically, they are used to transfer radiation from an extended source to a target, and are used as energy concentra-

Received 22 June 2004; accepted 5 September 2004

Correspondence to: J. Alda

Fax: ++34-91-394-6885

E-mail: j.alda@fis.ucm.es tors in solar furnaces. Passive solar tracking is also possible when spatial integrators are included in natural lighting systems [1].

Within the matrix optics framework [2, 3], the first design condition for a light integrator array is the condition for vanishing determinant. This condition is fulfilled when the distance $R$ between the input plane and synthetic image plane obeys the following formula

$$
R=t-\frac{b}{d} \text {. }
$$

Where $t$ is the length of the individual optical elements, and $b$ and $d$ are the elements of the matrix of each individual element of the array. Previous contributions have proposed some arrangements to improve the efficiency of these kinds of arrays [4-7], and make them easier to be integrated in architectural structures and natural lighting applications by allowing a plane configuration instead of the typical spheric dome setup [1], [8]. In this paper we focus our attention on spatial integrators having a plane arrangement and composed of individual units constructed with two thin lenses. We analyze the influence of the power of the thin lenses located at the input surface of the array, and the number of individual units, on the amount of transferred flux, and on the uniformity of the synthetic image plane.

In section 2 of this paper we present a theoretical analysis, based on matrix optics calculations, that relates the type of the optical element in the input surface of the array (diverging or converging), its optical power, and the maximum number of identical elements of the array that can work together in order to transfer the incident flux onto the synthetic image plane regardless the efficiency of each array element. These elements will be named as useful elements because at least one ray is able to be transferred from the first lens to the second one. In section 3 we have evaluated, by numeric calculations, several parameters that describe the performance of the spatial integrator as a function of the optical power value of the first lens, and their location in the optical array. Section 4 presents the principles of design that are applied to the 
simulation and comparison of two different thin lens planar spatial integrator arrays. Finally, section 5 summarizes the main conclusions of the paper.

\section{Plane spatial integrators. The two thin lenses design}

A scheme of a plane spatial integrator array constructed with units having two thin lenses is shown in fig. 1. It is composed of a pair of Planar Thin Lens Arrays (PTLA) separated by a distance $t$; the pitch of PTLA1, located at the input plane, is longer than that of PTLA2. This is necessary for the array to behave as a spatial integrator. Every individual element of the array is composed of a couple of thin lenses of different focal lengths $f_{1}$ and $f_{2}$. The optical axis of each element is the line joining the center of the thin lenses forming the unit. Each element has its own optical axis. All these optical axes intersect at the center of the synthetic image plane. It follows from equation (1) that, for a thin lens light integrator array, the distance between the input plane and the synthetic image plane of the array is,

$$
R=t\left(1-\frac{f_{2}}{f_{2}-t}\right) .
$$

The last equation indicates that the spatial integration feature of this type of arrays does not depend on the focal length of the first thin lens, $f_{1}$, which is left as a free parameter in the design of the array.

In a previous contribution the focal length values for PTLA1 optical elements are optimized to transfer every ray inciding on it to the synthetic image plane [7]. Another approach, analyzed in this paper, is to maximize the number of individual elements that can work together for a given value of $f_{1}$. In this case we

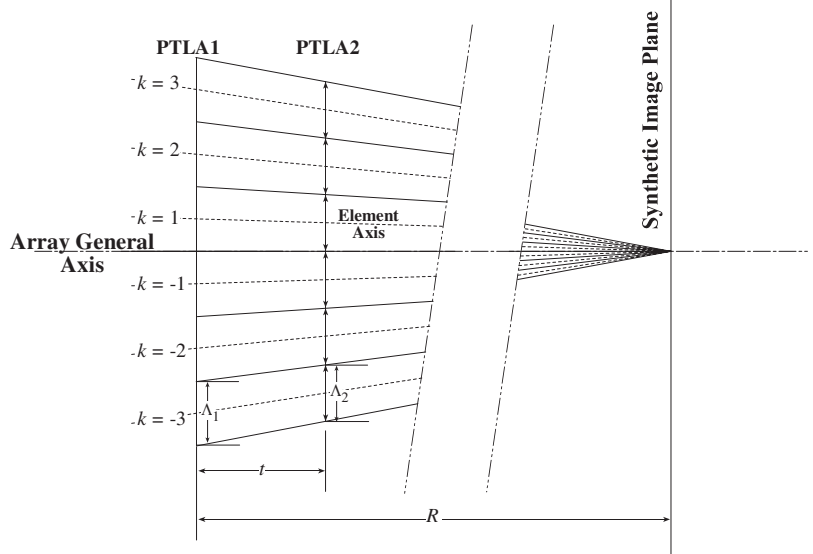

Fig. 1. Scheme of a light integrator array composed of a couple of plane thin lens arrays (PTLA1 and PTLA2). The geometrical parameters are given in table 1. consider that all the elements of PTLA1 have the same focal length, $f_{1}$. Then, we need to know how many elements transfer any portion of light rays incident to the synthetic image plane.

Fig. 1 represents the configuration of the spatial integrator along a medional plane when it is constructed with thin lenses. As the axis of the individual element becomes more and more misaligned, the efficiency of the elements to transfer radiation to the synthetic image plane becomes smaller. In the following calculations we evaluate the maximum number of useful elements that a plane thin lens spatial integrator array may have for two positive thin lenses and for negative thin lenses. We will consider an element to be useful when, at least, one of the rays incident on the input plane of the array, reaches the second lens, and therefore it is transferred to the synthetic image plane. All the calculations are done for a meridional plane. On the other hand, the spatial integrator has not a central element. The elements are arranged side by side, being the optical axis of the whole array the separation between contiguous elements of order +1 and -1 . In the following subsection we will calculate the limit conditions to obtain the maximum number of useful elements. Due to the symmetry of the arrangement we will only consider positive orders $(k>0)$.

\subsection{Positive lenses at the input plane}

In this case, the focal length of the elements at PTLA1 are converging lenses with the same focal length, $f_{1}$. At the same time, the elements of PTLA2 are of identical focal length, $f_{2}$, to comply with the vanishing determinant condition of equation (2). PTLA1 and PTLA2 are of different pitch: $\Lambda_{1}$ and $\Lambda_{2}$ respectively, being $\Lambda_{1}>\Lambda_{2}$.

Let us begin with positive PTLA1 having $f_{1}$ values greater than the individual element size $t$. The maximum order that the individual element can reach is defined by the ray incident at the lower extreme of the $k^{\text {th }}$ element of PTLA1, and after being deflected by the first lens, reaches the upper extreme of the element in PTLA2, (see fig. 2a). Considering a parallel bundle of rays also aligned with the optical axis of the array, this limit condition can be expressed in a matricial form as;

$$
\left(\begin{array}{c}
-\frac{\Lambda_{1}}{2} \\
\omega_{k}
\end{array}\right)=\left(\begin{array}{cc}
1 & 0 \\
-\frac{1}{f_{1}} & 1
\end{array}\right)\left(\begin{array}{c}
-\frac{\Lambda_{1}}{2} \\
0
\end{array}\right),
$$

where,

$$
\omega_{k}=\frac{\Lambda_{1}-k\left(\Lambda_{1}-\Lambda_{2}\right)}{t} .
$$

The maximum order of the element for $f_{1}>t$;

$$
k=\operatorname{Trunc}\left(\frac{\Lambda_{1}\left(2 f_{1}-t\right)}{2 f_{1}\left(\Lambda_{1}-\Lambda_{2}\right)}\right),
$$

where Trunc $(x)$ provides the integer part of $x$. 
PTLA1

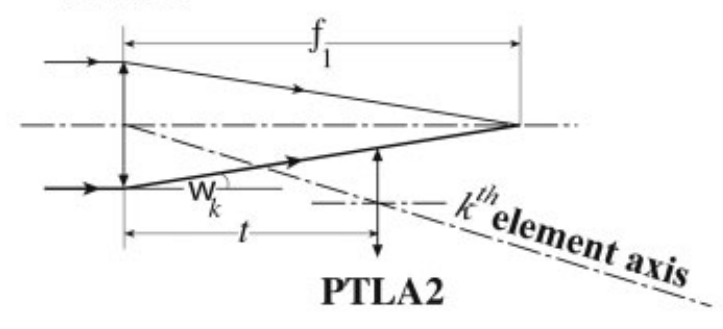

PTLA1
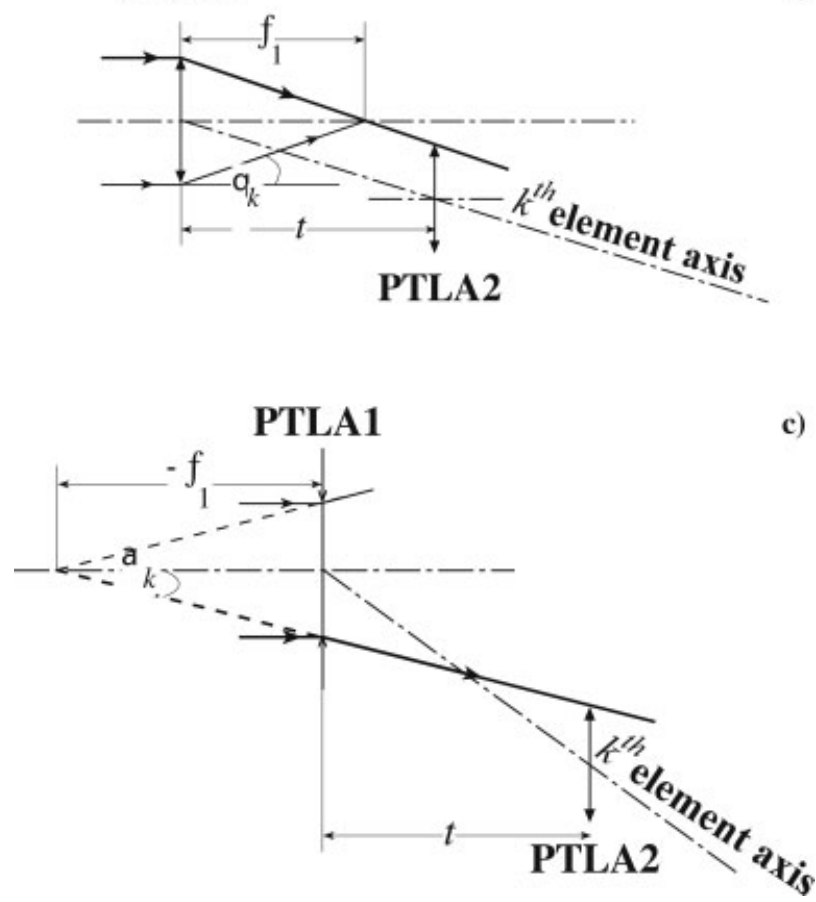

Fig. 2. Scheme of a light integrator array element composed of a couple of thin lenses, the output lens has a positive focal length $f_{2}$, on the other hand the input lens has. a) positive focal length $f_{1}>t$; b) positive focal length $f_{1}<t$; c) negative focal length.

A similar calculation can be made for positive PTLA1 with $f_{1}$ values smaller than the individual element size, $t$. The maximum order for the individual element can be calculated by considering the light ray incident at the maximum height of the $k^{\text {th }}$ element of PTLA1 to be deviated to an angle $\theta_{k}$, (see fig. 2b). Considering again a parallel bundle of rays aligned with the axis of the optical array, the condition to include the element $k^{\text {th }}$ as a useful element is written in matricial form as;

$$
\left(\begin{array}{c}
\frac{\Lambda_{1}}{2} \\
-\theta_{k}
\end{array}\right)=\left(\begin{array}{cc}
1 & 0 \\
-\frac{1}{f_{1}} & 1
\end{array}\right)\left(\begin{array}{l}
\frac{\Lambda_{1}}{2} \\
0
\end{array}\right)
$$

where,

$$
\theta_{k}=\frac{k\left(\Lambda_{1}-\Lambda_{2}\right)}{t} .
$$

The maximum order of the element for $f_{1}<t$;

$$
k=\operatorname{Trunc}\left(\frac{t \Lambda_{1}}{2 f_{1}\left(\Lambda_{1}-\Lambda_{2}\right)}\right) .
$$

\subsection{Negative lenses at the input plane}

b)

In this subsection we consider the case of having negative lenses at the PTLA1. Now the condition that defines the maximum number of useful elements of the array appears when a ray inciding on the $k^{\text {th }}$ element of PTLA1 at $-\frac{\Lambda_{1}}{2}$ is deviated to an angle $\alpha_{k}$, to reach the corresponding element on PTLA2, (see fig. 2c). Considering a parallel bundle of rays the matricial relation can be written as

$$
\left(\begin{array}{c}
-\frac{\Lambda_{1}}{2} \\
-\alpha_{k}
\end{array}\right)=\left(\begin{array}{cc}
1 & 0 \\
-\frac{1}{f_{1}} & 1
\end{array}\right)\left(\begin{array}{c}
-\frac{\Lambda_{1}}{2} \\
0
\end{array}\right),
$$

where

$$
\alpha_{k}=\frac{k\left(\Lambda_{1}-\Lambda_{2}\right)-\Lambda_{1}}{t} .
$$

The maximum order of the element is given by;

$$
k=\operatorname{Trunc}\left(\frac{\Lambda_{1}\left(t-2 f_{1}\right)}{2 f_{1}\left(\Lambda_{1}-\Lambda_{2}\right)}\right) .
$$

In all the previous calculations we should note that the maximum number of useful elements of the array is twice the maximum order of the element $k$. This is because the elements are arranged symmetrically around the optical array axis.

\section{Simulation and results}

In order to understand and clarify the meaning of the previously obtained relations, we have analyzed the behavior of thin lens spatial integrator arrays with maximum number of useful elements at different posi-

Table 1. Geometrical and optical parameters of the plane thin lens spatial integrator used in the computer simulation. The refractive index of the lenses material is $\mathbf{1 . 5 2 3}$.

\begin{tabular}{|c|c|}
\hline$R$ & $1280 \mathrm{~mm}$ \\
\hline$f_{2}$ & $137.9 \mathrm{~mm}$ \\
\hline$\Lambda_{1}$ & $80 \mathrm{~mm}$ \\
\hline$\Lambda_{2}$ & $70 \mathrm{~mm}$ \\
\hline$t$ & $160 \mathrm{~mm}$ \\
\hline
\end{tabular}




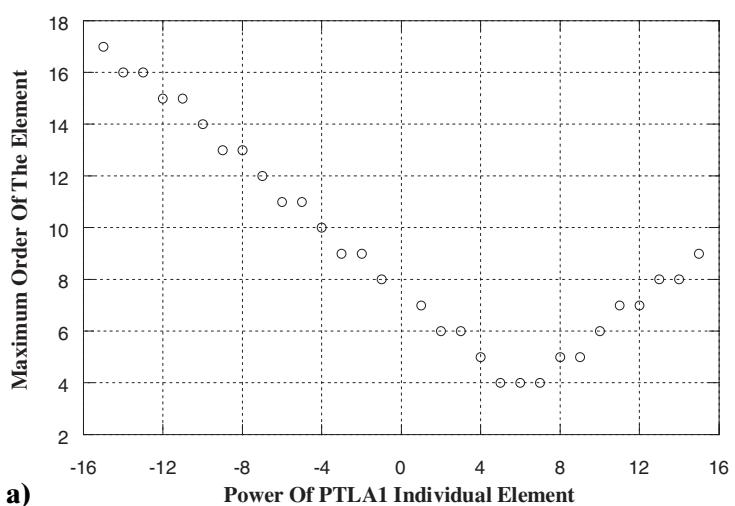

a)

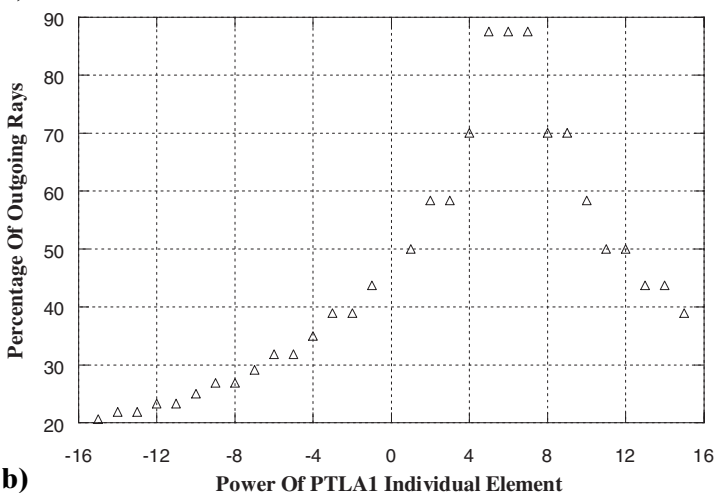

b)



c)

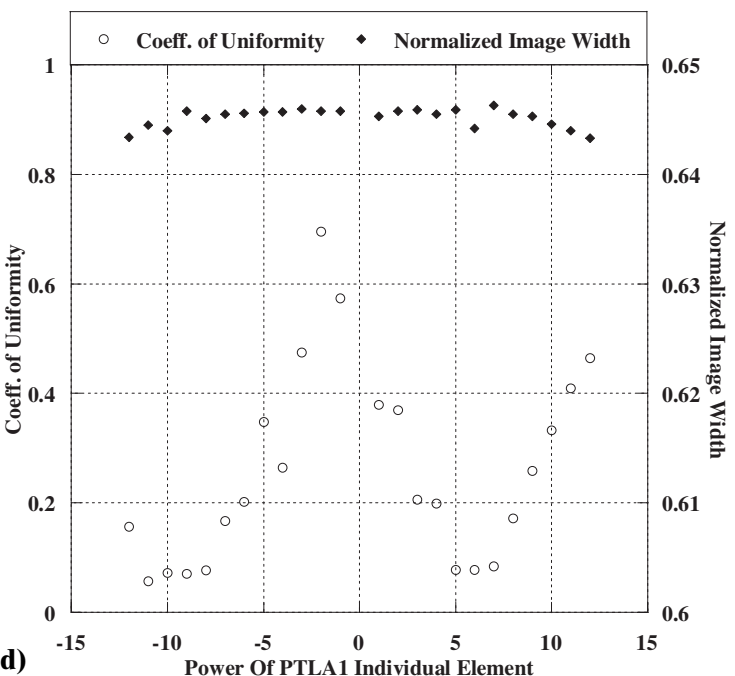

tive and negative PTLA1 power values. We considered an input planar thin lens array (PTLA1) of identical elements. A computer program has been developed to estimate the behavior of each array. The geometrical parameters of the array used in the simulation are given in table 1 (these values have been chosen after optimizing the focal length of the first PTLA, as it is presented in [7] and [8]). In fig. 3 we have plotted the several design and performance parameters as a function of the optical power of PTLA1 in diopters $(D)$ : (a) The maximum order of the last useful element obtained from equations (5), (8), and (11). (b) The percentage of the outgoing rays from each array with respect to the incoming rays. (c) The average transmittance of the array elements. (d) The image width $(\varpi)$ and the coefficient of uniformity $(u)$ for the energy distribution on the synthetic image plane, $\phi(x)$, defined as

$$
u=\int_{\text {paraxial }}\left|\frac{\partial \phi(x)}{\partial(x)}\right| \mathrm{d} x .
$$

This parameter is zero for a perfect uniform distribution within the paraxial synthetic image (note that the integration is taken only on the spatial region of the paraxial synthetic image plane). Although the uniformity of the image varies significantly with the power of the elements in PTLA1, the image size obtained from the numerical simulation changes slightly. This fact coincides with the theoretical predictions of the paraxial size of the synthetic image $\Delta$ given by [1],

$$
\Delta=\frac{\Lambda_{1}}{\left(1-\frac{t}{f_{2}}\right)} .
$$

From the plots of fig. 3 we may conclude that when the energy efficiency and the percentage of transferred rays increases, the number of useful elements decreases, and vice-versa. These dependences balance the performance of the design. However, as we will check later, different choices in the design may produce different behavior and different qualities of the synthetic image.

We have also calculated the efficiency of each individual element as a function of its location on the spatial integrator, and for several negative and positive values of the optical power of the first lens $F_{1}$. As well as the efficiency of each individual element, the percentage of outgoing rays with respect to the incoming ones, are

Fig. 3. These figures represents, as a function of the optical power of the first lens of the individual unit, the following parameters: a) maximum order of the useful element; b) percentage of outgoing rays with respect to incoming rays; c) average transmittance of all array elements; d) image width, $\varpi$, and coefficient of uniformity, $u$, for the energy distribution on the synthetic image plane. 

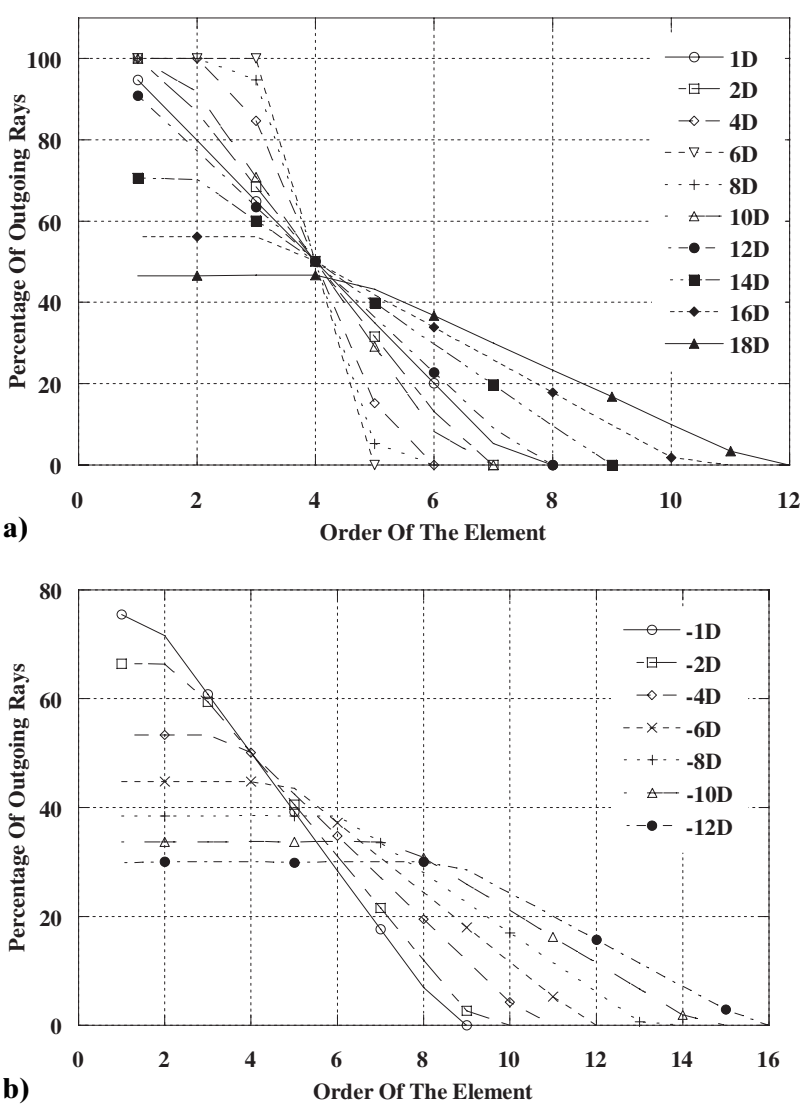

Fig. 4. Percentage of outgoing rays with respect to incoming rays as function of the individual unit order, for a) positive PTLA1 power values, and b) for negative PTLA1 power values.

calculated for positive and negative power values. The results are given in fig. 4 for positive focal lengths (a) and negative values (b).

The previous graphs allow the design of different spatial integrator arrays customized for different purposes. In other words, these graphs can be used as a guide for the design of spatial integrators, as it is illustrated in the following section.

\section{Design}

Spatial integrators may have different uses. Then, it is of interest to know what kind of array behaves the best for a given purpose. In order to clarify the design process we have itemized several parameters that can be of use when defining the global behavior of the array. On one side we have the constructive parameters: number of useful elements in the spatial integrator, and focal length of the first lenses. On the other hand we will check the synthetic image characteristics. They are described by the uniformity of the synthetic image and by the efficiency in the transfer of flux.
In the following, we will analyze of two different plane spatial integrators designs constructed with thin lenses. The first spatial integrator array has been designed having a maximum number of identical elements on PTLA1. It maximizes the transmittance, and the number of outgoing rays with respect to the number of incoming rays. Besides, the width of the synthetic image is close to the paraxial size, and its uniformity and energy efficiency is optimized (see fig. 3). In this array, the PTLA1 has 8 optical elements (the maximum order is $k=4$ ), and every one has the same optical power equal to $5 \mathrm{D}$. In the second array the elements in PTLA1 are not identical. It has been designed to transfer as much as possible energy onto the synthetic image plane regardless its uniformity. For each optical element in PTLA1, we have considered its individual behavior at a given position (order) with a definite power. Referring to fig. 4, the thin lens elements in PTLA1 were chosen to have the powers given in table 2 , corresponding to each element order. The maximum order of the element in this case is $k=11$. As the optical elements are arranged on both sides of the array general axis, the total number of elements for this array is 22 . Three factors were taken into account in designing such array. First, we increased the maximum number of elements (array area) that can work together to produce the synthetic image. Second, we were looking to obtain the maximum efficiency. And finally, we prefer, when possible, large focal length elements on PTLA1, as they will be cheaper and will behave better because their F\# will not be too low.

Table 2. Power (in diopters) of the thin lens elements in PTLA1 corresponding to each order. Case (a): spatial integrator with maximum number of identical elements. Case (b): spatial integrator having elements with different optical characteristics.

\begin{tabular}{|c|c|c|}
\hline$k \#$ & $\begin{array}{c}\text { Case (a) } \\
\text { Power at PTLA1 } \\
\left(\text { in }^{-1}\right)\end{array}$ & $\begin{array}{c}\text { Case (b) } \\
\text { Power at PTL1 } \\
\left.\text { (in m }^{-1}\right)\end{array}$ \\
\hline 1 & 5 & 2 \\
\hline 2 & 5 & 4 \\
\hline 3 & 5 & 5 \\
\hline 4 & 5 & -6 \\
\hline 5 & - & -6 \\
\hline 6 & - & -6 \\
\hline 7 & - & -6 \\
\hline 8 & - & -6 \\
\hline 9 & - & -6 \\
\hline 10 & - & -6 \\
\hline 11 & - & \\
\hline
\end{tabular}




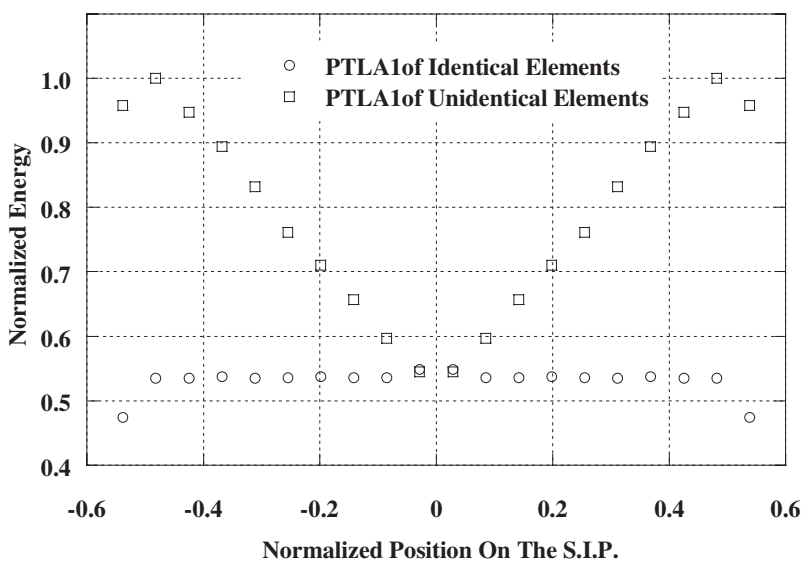

Fig. 5. Energy distribution on the synthetic image plane (SIP) normalized to the maximum value for two different designs of a plane thin lens spatial integrator array. The squares correspond to PTLA1 with different elements and the circles represent the array with PTLA1 of identical elements.

In order to compare the behavior of the two previously designed light integrator arrays, we have calculated the energy distribution on the synthetic image plane (see fig. 5). The position is normalized to the paraxial size of the image $\Delta$. Also we have calculated the coefficient of uniformity (within the image paraxial size) corresponding to each energy distribution, and the percentage of the outgoing rays and energy reaching the synthetic image plane. All these values are presented in table 3 .

Table 3. Coefficient of uniformity of the energy distribution (the lower the value the better uniformity), energy reaching the synthetic image plane (in arbitrary units) and ratio (in percentage) between the number of outgoing rays and the number of incoming rays in case of: (a) plane thin lens light integrator array of identical elements with parameters given in table (1) and PTLA1 elements with powers given in table (2). Case (b): corresponds to a planer light integrator array of unidentical elements with parameters given in table (1) and PTLA1 elements with powers given in table (2).

\begin{tabular}{|c|c|c|c|}
\hline & $\begin{array}{c}\text { Coeff. Of } \\
\text { uniformity, } u\end{array}$ & $\begin{array}{c}\text { Energy on the } \\
\text { Synthetic image plane } \\
\text { (A.U.) }\end{array}$ & $\begin{array}{c}\text { Percentage } \\
\text { of outgoing } \\
\text { rays }\end{array}$ \\
\hline Case (a) & 0.0769 & 4690.1 & 75 \\
\hline Case (b) & 0.9097 & 6977.4 & 47.35 \\
\hline
\end{tabular}

\section{Conclusions}

By studying the behavior of light integrator arrays formed by a couple of plane thin lens arrays, we have found how the power of the lenses located at the input array influences important constructive and performance parameters. In particular, we have analyzed the maximum number of useful elements for a given angular condition. This number limits the lateral size actually contributing to the synthetic image. When all the optical elements at the input plane are equal, the focal length of the first thin lens also changes the efficiency of the array represented by the amount of energy and the percentage of outgoing rays with respect to incoming energy and number of rays incident on the synthetic image plane. Finally, the quality of the synthetic image, parametrized by its width and its uniformity coefficient, has been also evaluated as a function of the optical power of the first lens. The significance of the previously obtained results has helped us to design two different kinds of planar thin lens light integrators adequate for specific array applications. A comparative analysis of the result has shown how the choice of different parameters of design changes the global behavior of the spatial integrator, allowing a customization of the array.

Acknowledgements. This work has been partially supported by the project TIC2001-1259 from the Ministerio de Ciencia y Tecnología of Spain.

\section{References}

[1] Vázquez D, Bernabéu E: Array optical devices for natural lighting. Light Res. Technol. 29 (1997) 33-39

[2] Wang S, Ronchi L: Principles and design of optical arrays. In Wolf E (Ed.): Progress in Optics. 25 (1988) 279-347

[3] Wang S, Zhao D: Matrix optics. Springer-Verlag, Heidelberg 2000

[4] Alda J, Kamal H, Bernabéu E: Optimum design of optical arrays with spatial integration feature. Opt. Eng. 36 (1997) $2872-2877$

[5] Kamal H: Design and properties of optical arrays. PhD dissertation, Universidad Complutense de Madrid, Spain 1998

[6] Kamal H: Optimization of the input surface of a thick-lens spatial-integrator array for maximum flux transfer efficiency. Optik 114 (2003) 213-216

[7] Kamal H, Vázquez D, Alda J: Optimum focal length range for thin-lenses optical arrays having spatial integration feature (submitted to Optical Engineering, 2004)

[8] Vázquez D, Alda J, Bernabéu E: Planar optical array with a spatial integration feature. Appl. Opt. 38 (1999) $1133-$ 1138 